\title{
Generalized Apollonian Packings
}

\author{
Daniel Bessis ${ }^{1}$ and Stephen Demko ${ }^{2}$ \\ ${ }^{1}$ Service de Physique Théorique ${ }^{\star}$ de Saclay, F-91191 Gif-sur-Yvette Cedex, France \\ 2 School of Mathematics, Georgia Institute of Technology, Atlanta, Georgia 30332, USA
}

Received March 21, 1990

\begin{abstract}
In this paper we generalize the classical two-dimensional Apollonian packing of circles to the case where the circles are no more tangent. We introduce two elements of $S L(2, \mathbb{C})$ as generators: $R$ and $T$ that are hyperbolic rotations of $\frac{2 \pi}{3}$ and $\frac{2 \pi}{N}(N=2,3,4, \ldots)$, around two distinct points. The limit set of the discrete group generated by $R$ and $T$ provides, for $N=7,8,9, \ldots$ a generalization of the Apollonian packing (which is itself recovered for $N=\infty$ ). The values $N=2,3,4,5$ produce a very different result, giving rise to the rotation groups of the cube for $N=2$ and 4 , and the icosahedron for $N=3$ and 5 . For $N=6$ the group is no longer discrete. To further analyze this structure for $N \geqq 7$, we move to the Minkowski space in which the group acts on a one sheeted hyperboloid. The circles are now represented by points on this variety and generate a crystal on it.
\end{abstract}

\section{Introduction}

In a classical construction of an Appolonian packing, one starts with a curvilinear triangle and constructs the inscribed circle, thus creating three curvilinear triangles out of the original one. This process is then repeated with each of the resulting curvilinear triangles and their descendants. The method for producing the inscribed circles can be realized with inversions [1] or Möbius maps [2].

In this latter case, one sees that the Apollonian packing is the limit set of a discrete subgroup of $S L(2, \mathbb{C})$. We present here a technique for generating nontangential disk packings as limit sets of discrete groups which include as a special case the Apollonian packing. Extension to higher dimensional sphere packings will be presented elsewhere.

Disk and sphere packings are natural models for porous media $[3,4]$ and a

\footnotetext{
* Laboratoire de l'Institut de Recherche Fondamentale du Commissariat à l'Energie Atomique
} 
parametrized family of such packings would be a valuable tool for making models of various media having different porosities. The group invariance would be useful in the study of flows in such models. These considerations, prompted by the experimental results of [5] were the motivation for our initial investigations.

More recently, the fact that the computer pictures of our packings bear some resemblance to $X$-ray scatter plots of crystals and quasi-crystals [6] has lead us to wonder if there might be some connection between our work and these areas. The family of groups related to our packings contains the finite groups of symmetries of the cube and icosahedron; so there is an (unexpected) relation to crystals. In addition, the typical fractals generated by our infinite group only realize their total symmetry in the limiting process. This is somewhat akin to the long-range symmetry of quasi-crystals [7]. Furthermore the fact that there is a one-to-one correspondence between these fractals and a regular lattice drawn on a one sheeted space-like hyperboloid may give some support to these considerations.

The paper is organized as follows:

In Sect. II, we give the basic geometric construction and derive the related family of groups which depends on a parameter $\alpha$. We show that the group can be generated by two rotations, one of $2 \pi / 3$ and one of $2 \alpha$, and that the group always contains the alternating group on four elements. In Sect. III we study the disk packing aspects. We prove that $\alpha=\frac{\pi}{N}$ for $N \in \mathbb{N}$,
and $N \geqq 7$ is a necessary condition to have a packing.

In Sects. IV and V, we consider a representation of our groups as groups of isometries on a four-dimensional Minkowski space and represent the action of the group in terms of Chebyshev polynomials.

In Sects. VI and VII, we study the case $\alpha=\pi / N$ for $2 \leqq N \leqq 5$ and $N=6$ respectively. In the former case, we obtain the group of the cube and icosehadron; in the latter case the group is not discrete.

In Sect. VIII, we indicate higher dimensional extensions.

\section{The Basic Construction}

Consider three circles with equal radii $r$ and centers at cubic roots of unity: $1, \omega, \omega^{2}$. Label the circles $X_{1}, X_{2}, X_{3}$ in counterclockwise order starting with the one whose center is at 1 . We seek a fourth circle $X_{0}$ and three Möbius maps $T_{1}, T_{2}, T_{3}$, such that

$$
\begin{gathered}
T_{i} X_{j}=X_{j}, \quad j \neq i, \quad i, j=1,2,3, \\
T_{i} X_{i}=X_{0} \\
T_{2}=R T_{1} R^{-1} \\
T_{3}=R^{-1} T_{1} R
\end{gathered}
$$

where $R$ is a rotation of $+\frac{2 \pi}{3}$ around the origin. It follows that $X_{0}$ will have center at 0 because it is invariant under $R$ :

$$
R X_{0}=R T_{1} X_{1}=R T_{1} R^{-1} R X_{1}=T_{2} R X_{1}=T_{2} X_{2}=X_{0}
$$


One can construct $T_{1}$ as a composition:

$$
T_{1}=S J
$$

where $S$ is the reflection through the axis of symmetry of $X_{2}$ and $X_{3}$ and $J$ is the inversion with respect to a circle orthogonal to $X_{2}$ and $X_{3}$. Some elementary computations (see Appendix A) reveal that

$$
T_{1} z=\frac{(I-1)}{2} \frac{2 z-I}{z+I}
$$

where $I$ is the center of the circle of inversion:

$$
I=\frac{1+\sqrt{9-8 r^{2}}}{2} \text {. }
$$

The fixed points of $T_{1}$ are the points of intersection of the inversion circle $J$ with the axis of symmetry of $X_{2}$ and $X_{3}$. These are $f$ and $f^{*}$ (see Fig. 1),

$$
f=-\frac{1}{2}+i \beta
$$

where

$$
\beta=\sqrt{\frac{3}{4}-r^{2}}=\frac{1}{2} \frac{\sin \alpha}{\sqrt{\cos ^{2} \alpha-2 / 3}} .
$$

For the moment we shall consider that $r$ vary from zero where all $X_{i}$ are reduced to points, to $r_{\max }=\frac{\sqrt{3}}{2}$, where all $X_{i}$ are tangent. In this range of values of $r$, no $X_{i}$ intersect (except for $r=r_{\max }$ ).

The angle $\alpha$ in Fig. 1 which is the argument of $(f+I)$ is defined by:

$$
\cos \alpha=\frac{I-\frac{1}{2}}{k}
$$

or in terms of $r$ :

$$
\cos \alpha=\frac{1}{2} \sqrt{\frac{9-8 r^{2}}{3\left(1-r^{2}\right)}} \text { or } \quad r^{2}=\frac{3}{2} \frac{1-2 \cos 2 \alpha}{1-3 \cos 2 \alpha} .
$$

The radius $\rho_{0}$ of the circle $X_{0}$ (of center 0 ) is easily computed (see Appendix A). We also introduce the circle $X_{\infty}$ defined by:

$$
X_{\infty}=T_{1}^{-1} X_{1} \text {. }
$$

One checks that the center of $X_{\infty}$ is 0 . Therefore $X_{\infty}$ is invariant under $R$ and we see that:

$$
X_{\infty}=T_{i}^{-1} X_{i}, \quad i=1,2,3 .
$$

The radius $\rho_{\infty}$ of $\left(X_{\infty}\right)$ is given in Appendix A. In particular we have:

$$
\rho_{0} \rho_{\infty}=1-r^{2}=\frac{1}{2(3 \cos 2 \alpha-1)} .
$$




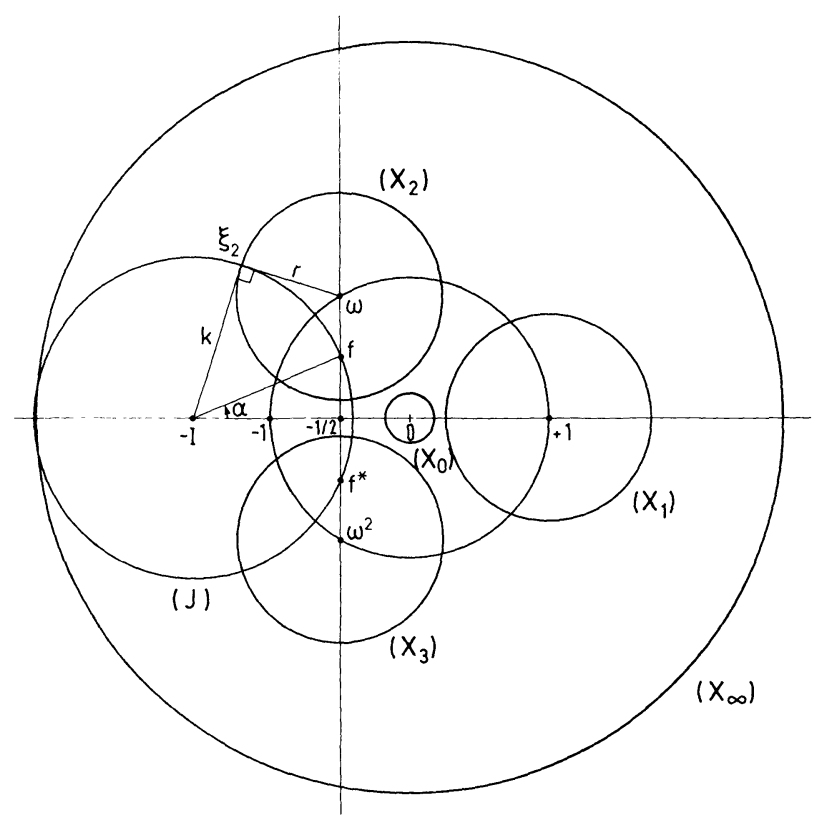

Fig. 1. The basic construction. $\alpha=\pi / 8, r=0.744, I=1.568, k=1.157, \rho_{0}=0.164, \rho_{\infty}=2.706$

For the reader's convenience, we recall that a Möbius transformation:

$$
T=\left[\begin{array}{ll}
a & b \\
c & d
\end{array}\right] \text { with } a d-b c=1
$$

transforms the circle of center $\gamma$ and radius $r$ into a circle of center $\gamma^{\prime}$ and radius $r^{\prime}$ with

$$
\begin{aligned}
& \gamma^{\prime}=\frac{a}{c}-\frac{1}{c} \frac{1}{(c \gamma+d)} \frac{1}{1-\frac{r^{2}}{\left|\gamma+\frac{d}{c}\right|^{2}}} \\
& r^{\prime}=\frac{r}{|| c \gamma+\left.d\right|^{2}-r^{2}|c|^{2}}
\end{aligned}
$$

Equation (II.16) allows to check that the center of $X_{\infty}$ is 0 . In particular we have

$$
T_{1}=\left[\begin{array}{cc}
\frac{2(I-1)}{\sqrt{6 I(I-1)}} & \frac{-I(I-1)}{\sqrt{6 I(I-1)}} \\
\frac{2}{\sqrt{6 I(I-1)}} & \frac{2 I}{\sqrt{6 I(I-1)}}
\end{array}\right]=\left[\begin{array}{cc}
\cos \alpha-\sqrt{\cos ^{2} \alpha-2 / 3} & -\frac{1}{6} \frac{1}{\sqrt{\cos ^{2} \alpha-2 / 3}} \\
2 \sqrt{\cos ^{2} \alpha-2 / 3} & \cos \alpha+\sqrt{\cos ^{2} \alpha-2 / 3}
\end{array}\right] .
$$


We notice that $T_{1}^{-1}$ is given by the same matrix, choosing the other determination in the square root of (II.7). Therefore, the other determination generates the same group, with $X_{0}$ and $X_{\infty}$ interchanged.

Mobiüs transformations in the form of(II.15) are commonly classified according to their trace: $\operatorname{Tr} T=a+c,[8]$. We are interested in the values of $r$ that make $T_{1}$ be either parabolic $\left\{\left(\operatorname{Tr} T_{1}\right)^{2}=4\right\}$ or elliptic $\left\{\left(\operatorname{Tr} T_{1}\right)^{2} \in[0,4)\right\}$ in which case $T_{1}$ is a rotation by $2 \alpha$. We have the following:

Proposition 1. $T_{1}$ is parabolic if and only if $r^{2}=3 / 4 . T_{1}$ is elliptic if and only if either $r^{2} \geqq 9 / 8$ or $r^{2}<3 / 4$.

In the range $0 \leqq r^{2} \leqq 3 / 4, \alpha$ decreases from $\frac{\pi}{6}$ to 0 . The case $r^{2}=3 / 4$ corresponds to mutually tangent circles $X_{1}, X_{2}, X_{3}$ with $X_{0}$ the inscribed circle and $X_{\infty}$ the circumscribed circle. The images of $X_{0}$ under the members of the group produce the classical Apollonian packing that has been investigated and extensively studied, $[1,9,10,11]$. In the cases $0 \leqq r^{2}<3 / 4$ it is necessary that the $T_{i}$ 's generate a discrete group if we are to obtain a packing from the image of $X_{0}$ under the group elements. In these cases $2 \alpha$, the angle of rotation, should be a rational multiple of $2 \pi$. That is, a necessary condition to have a packing is

$$
\alpha=\frac{k \pi}{N}, \quad k, N \in \mathbb{N}
$$

We shall prove later on, that for $0 \leqq r^{2} \leqq \frac{3}{4}$, it is necessary to choose $k=1$, otherwise the orbit of $X_{1}$ under the cyclic group generated by $T_{1}$ will consist of overlapping circles. Also in this case $N$ is necessarily at least 7 . In any case we shall restrict our discussion to

$$
\alpha=\frac{\pi}{N}
$$

and distinguish two cases: (i) $N=2,3,4,5,6$. (ii) $N \geqq 7$. In case (ii) to be discussed in the next section, the limit set of the group generated by $T_{1}, T_{2}, T_{3}$ appears to consist of infinitely many non-overlapping circles which form a packing of $X_{\infty}$ the common image of $T_{i}^{-1} X_{i}$. In case (i) the geometry of the circles $\left\{X_{i}\right\}$ does not play a central role. We note here a common feature of all cases.

Proposition 2. $T_{i} T_{j}^{-1}$ generate an isomorphic copy of $A_{4}$, the alternating group on 4 elements.

Proof. From the fact that

$$
T_{i}^{-1} X_{i}=T_{j}^{-1} X_{j}=X_{\infty} \text { for } 0 \leqq r \leqq \frac{\sqrt{3}}{2}
$$

one checks immediately that $T_{i} T_{j}^{-1}$ acts for $i \neq j$ as a cyclic permutation on $X_{i}, X_{j}, X_{0}$ and leaves the remaining circle invariant. The group relation implied by this being analytic in $r$, will remain true for any complex value of $r$. From:

$$
\begin{gathered}
T_{i} T_{j}^{-1} X_{i}=X_{0} ; \quad T_{i} T_{j}^{-1} X_{j}=X_{i} ; \quad T_{i} T_{j}^{-1} X_{0}=X_{j} ; \quad T_{i} T_{j}^{-1} X_{k}=X_{k} ; \\
i \neq j, \quad\{i, j=1,2,3\} .
\end{gathered}
$$


we see that the group generated by $T_{i} T_{j}^{-1}$ is isomorphic to $A_{4}$ and:

$$
\begin{aligned}
& a=T_{1} T_{2}^{-1}=(1,0,2)(3), \\
& b=T_{3} T_{2}^{-1}=(2,3,0)(1), \\
& c=T_{1} T_{3}^{-1}=(1,0,3)(2) .
\end{aligned}
$$

The twelve elements of the group isomorphic to $A_{4}$ are:

$$
\left\{I, a, a^{2}, b, b^{2}, c, c^{2}, a b, b a, c a, b c, a c^{2}\right\} \approx A_{4} .
$$

Here explicitly, we have

$$
\begin{aligned}
& a=(1,0,2)(3) ; a^{2}=a^{-1}=(2,0,1)(3) ; \quad b=(2,3,0)(1) ; \quad b^{2}=b^{-1}=(0,3,2)(1), \\
& c=(1,0,3)(2) ; c^{2}=c^{-1}=(3,0,1)(2) ; \quad a b=(1,0)(3,2) ; \quad b a=(3,0)(2,1), \\
& c a=(3,1)(2,0) ; \quad a c=b a ; \quad b c=(2,3,1)(0) ; \quad c b=a ; \quad a^{2} c=(3,1,2)(0) . \quad(\text { II. } .23) \\
& a b c=b .
\end{aligned}
$$

It follows that $R$ corresponds to $b c$. Therefore the elements of order 2 of the group are $a b, b a, c a$ and the $T_{i} T_{j}^{-1}$ are order 3 elements. One can construct easily the multiplication table of this group.

Proposition 3. Similarly $T_{i}^{-1} T_{j}$ generate an isomorphic copy of $A_{4}$.

Proof. Replace $X_{0}$ by $X_{\infty}$ everywhere in (II.20).

We shall denote by $\left\langle T_{1}, T_{2}, T_{3}\right\rangle$ the group generated by $T_{1}, T_{2}, T_{3}$. We notice that $T_{i} T_{j}^{-1}$ or $\left(T_{i}^{-1} T_{j}\right)$ is a finite subgroup of \langle\rangle , isomorphic to $A_{4}$ which is the group of symmetries of the tetrahedron. This remark will be useful later on.

\section{Packing Aspects}

In this section, we shall consider the case where $0 \leqq r<\frac{\sqrt{3}}{2}, T_{1}$ is elliptic. If we
want the group to be discrete, $\alpha$ should be of the form

$$
\alpha=\frac{k \pi}{N} .
$$

If we want the images of $X_{1}$ under the successive powers of $T_{1}$ not to overlap then necessarily $N \geqq 7$ and $k=1$,

$$
\alpha=\frac{\pi}{N}, \quad N=7,8,9, \ldots
$$

To prove statement (III.1) we need several steps which are outlined in the following.

To study more conveniently the action of $T_{1}$ on $X_{1}$, we have to move to a system of coordinates in which $T_{1}$ is diagonal. The diagonalized form of $T_{1}$ reads:

$$
D=\left(\begin{array}{cc}
e^{i \alpha} & 0 \\
0 & e^{-i \alpha}
\end{array}\right)
$$

Let $\Lambda$ be the transformation which diagonalizes $T_{1}$ :

$$
T_{1}=\Lambda D \Lambda^{-1} \text {. }
$$


With $f$ and $f^{*}$ (II.8) being the fixed points of $T_{1}$, we have

$$
\begin{aligned}
\Lambda(z) & =\frac{f z+f^{*}}{z+1}, \\
\Lambda^{-1}(z) & =-\frac{z-f^{*}}{z-f} .
\end{aligned}
$$

The study of the maps of $X_{1}$ under the various powers of $T_{1}$ is therefore equivalent to the study of the maps of $\Lambda^{-1} X_{1}$ under the powers of $D$.

Using formulae (II.16), and (II.8), (II.9), we find the center $\tilde{\gamma}$ and radius $\tilde{r}$ of $\Lambda^{-1} X_{1}$ to be

$$
\begin{aligned}
& \tilde{\gamma}_{1}=-\frac{3}{3-2 r^{2}}\left(\frac{1}{2}+i \sqrt{\frac{3}{4}-r^{2}}\right) ; \quad\left|\tilde{\gamma}_{1}\right|=\frac{3}{3-2 r^{2}} \sqrt{1-r^{2}}=\frac{\sqrt{3 \cos 2 \alpha-1}}{\sqrt{2 \cos 2 \alpha}} \\
& \tilde{r}_{1}=\frac{r \sqrt{3-4 r^{2}}}{3-r^{2}}=\frac{\sin \alpha}{\cos 2 \alpha} \sqrt{2 \cos 2 \alpha-1}
\end{aligned}
$$

$D$ rotates $\Lambda^{-1} X_{1}=\tilde{X}_{1}$ by $2 \alpha$. From $\tilde{0}_{1}$ the center of rotation of $D$, one sees the circle $\widetilde{X}_{1}$ under an angle $2 \phi$ :

or, using (II.14):

$$
\sin \phi=\frac{\tilde{r}_{1}}{\left|\tilde{\gamma}_{1}\right|}=\frac{r}{3} \sqrt{\frac{3-4 r^{2}}{1-r^{2}}}
$$

$$
\sin \phi=\sin \alpha \sqrt{\frac{2(2 \cos 2 \alpha-1)}{3 \cos 2 \alpha-1}}
$$

$\alpha$ being of the form $\frac{k}{N} \pi$ ( $k$ and $N$ relatively prime), the rotation $D$ defines exactly $N$ angular sectors of angle $\frac{2 \pi}{N}$ (see Fig. 2). If we want the various images of $\tilde{X}_{1}$ under $D$ not to overlap, it is necessary that $\tilde{X}_{1}$ be entirely contained in one of these sectors, that is:

$$
0<2 \phi<\frac{2 \pi}{N}
$$
We remark that $N$ has to be $\geqq 7$ in order for $\alpha$ to be $<\frac{\pi}{6}$. Then (III.8) implies,
using (III.7):

and

$$
\sin \frac{k \pi}{N} \sqrt{\frac{2\left(2 \cos \frac{2 k \pi}{N}-1\right)}{3 \cos \frac{2 k \pi}{N}-1}}<\sin \frac{\pi}{N},
$$

$$
0 \leqq \frac{k \pi}{N}<\frac{\pi}{6} .
$$




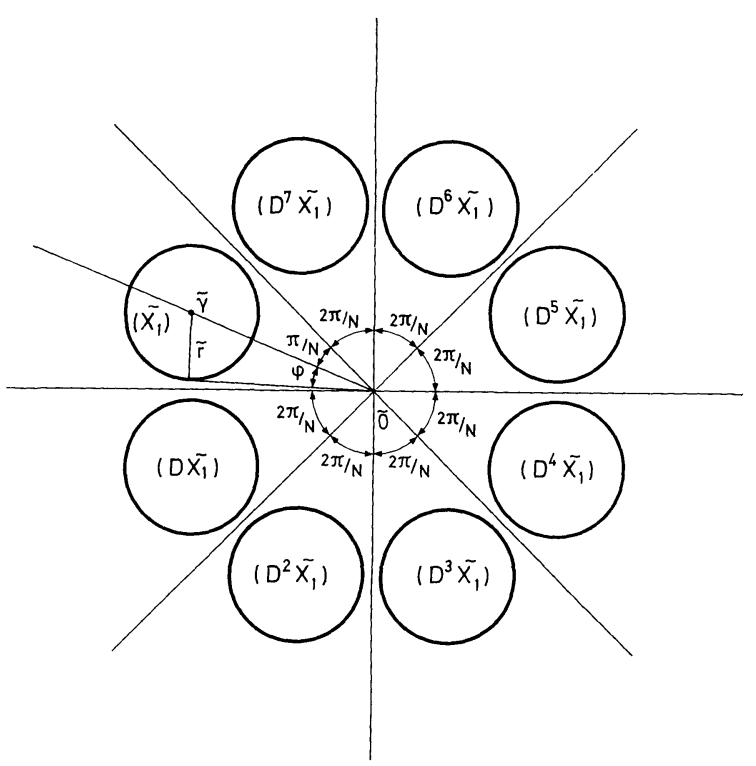

Fig. 2. The action of $D$ on $\tilde{X}_{1}$ for $N=8$

From (III.11) we see that

$$
\frac{\pi}{N}<\frac{\pi}{6 k} \quad \text { which implies } \quad \frac{\pi}{N} \leqq \frac{\pi}{6 k+1} .
$$

If we square (III.9) we get

$$
\frac{1-4 \sin ^{2} \frac{k \pi}{N} \sin ^{2} \frac{k \pi}{N}}{1-3 \sin ^{2} \frac{k \pi}{N} \sin ^{2} \frac{\pi}{N}}<1
$$

with

$$
\frac{\pi}{N} \leqq \frac{\pi}{6 k+1} .
$$

Equation (III.13) implies that $k=1$. The proof is a consequence of the following lemma.

Lemma. For $k \geqq 2$ and $0 \leqq \xi \leqq \frac{\pi}{6 k+1}, k \in \mathbb{N}$, we have

$$
\frac{1-4 \sin ^{2} k \xi}{1-3 \sin ^{2} k \xi} \cdot \frac{\sin ^{2} k \xi}{\sin ^{2} \xi}>1 .
$$

The proof of this lemma can be found in Appendix B. 


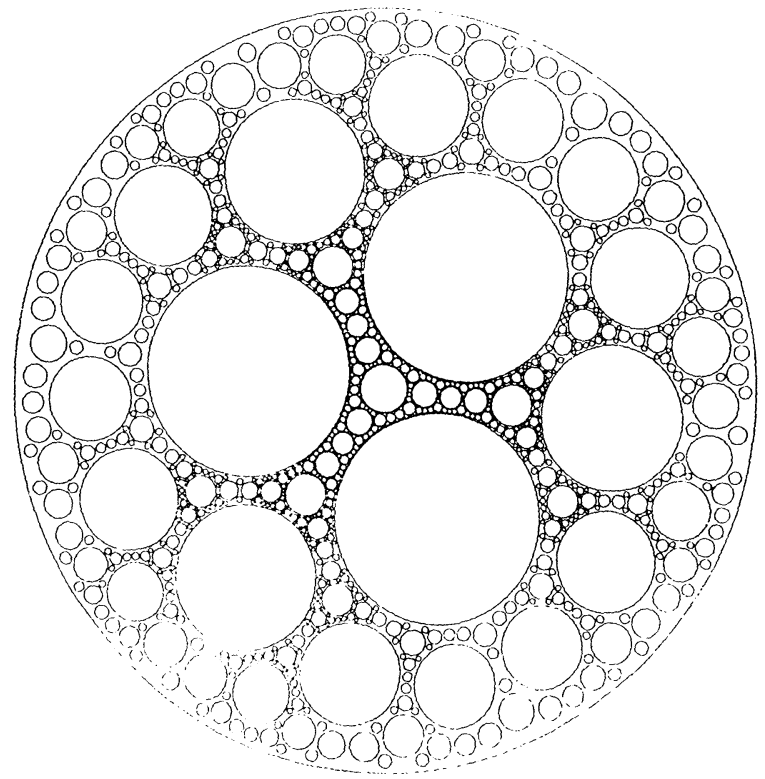

Fig. 3. The limit set for $N=8$

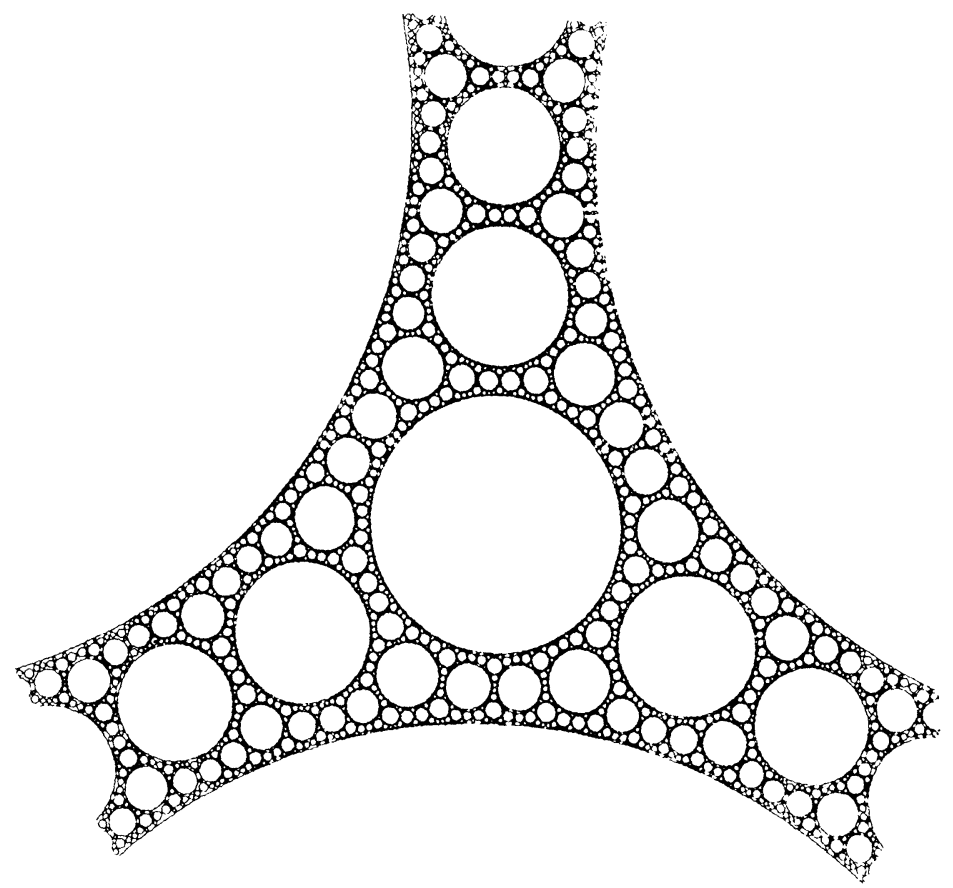

Fig. 4. A blow-up of Fig. 3 
$\pi$ We have performed computer graphical experiments with $\alpha$ of the form $\frac{\pi}{N}, N \geqq 7$, and constructed the successive maps of $X_{1}$ under the full group $\left\langle T_{1}, T_{2}, T_{3}\right\rangle$ see Figs. 3 and 4 , we have never observed overlapping of circles. However we presently have no proof of the following conjectures, although some hints toward a proof will be discussed in the later section.

Conjecture 1. For $\alpha=\frac{\pi}{N}, N \geqq 7$, the circles obtained by applying the full group $\left\langle T_{1}, T_{2}, T_{3}\right\rangle$ to $X_{1}$ do not cross each other, and each circle which is obtained an infinite number of times remains inside $X_{\infty}$. We therefore obtain a packing of $X_{\infty}$.

Conjecture 2. For $\alpha=\frac{k \pi}{N}, k \geqq 2(k, N$ relatively prime) the circles obtained in the above fashion form a nowhere dense subset of $\mathbb{C}$. That is $\left\langle T_{1} T_{2} T_{3}\right\rangle$ is discontinuous.

\section{Polyspherical Representations}

In order to try to prove that our circles do not overlap and to gain further insight into our problem, we introduce polyspherical coordinates for our circles. A more precise name would be tetracircular coordinates. An introduction to this subject can be found in [12 or 13].

IV.1. Notations and Definitions. $S(a, r)$ denotes the inner part of the circle of center $a$ and radius $r$ if $r>0$; if $r<0$ it will represent the outer part. Let

$$
X=S(a, r), \quad Y=S(b, s) .
$$

Then the "distance" between $X$ and $Y$ is

In particular

$$
\Delta(X, Y)=\frac{|a-b|^{2}-r^{2}-s^{2}}{2 r s} .
$$

$$
\begin{aligned}
\Delta(X, X) & =-1, \\
|\Delta(X, Y)| & >1
\end{aligned}
$$

if $X$ and $Y$ do not intersect. $|\Delta(X, Y)|<1$ means that the two circles $X$ and $Y$ intersect and the angle of intersection is:

$$
\cos \theta=-\Delta(X, Y) \text {. }
$$

The circles are oriented clockwise when $r>0$ and anticlockwise when $r<0$. The tangent are oriented accordingly, and $\theta$ is the supplement of the angle between the two oriented tangents. Given four circles $Z_{1}, Z_{2}, Z_{3}, Z_{0}$ in the plane, one defines the matrix $G$ according to:

$$
G^{i j}=\Delta\left(Z_{i}, Z_{j}\right), \quad i, j=0,1,2,3 .
$$

This is a real $4 \times 4$ symmetric matrix. If

$$
\operatorname{det} G \neq 0
$$


the matrix is invertible, and the four circles $X_{i}$ are said to be independent.

Let us define the contravariant coordinates of a circle $Y$ by:

$$
y^{i}=\Delta\left(Y, Z_{i}\right) \quad i=0,1,2,3 .
$$

The "polyspherical coordinate of $Y$ " will be the covariant components of $Y$, using $G^{i j}$ as metric tensor,

$$
y_{i}=\sum_{j=0}^{j=3} G_{i j} y^{j}=G_{i j} y^{j} \quad \text { with } \quad G_{i j} G^{j k}=\delta_{i}^{k} .
$$

(We use the convention of summation over repeated indices, when in upper and lower position.)

Notation. For simplicity we shall denote by $Y_{c}$ the set of covariant components of $(Y)$ :

$$
Y_{c}=\left(y_{0}, y_{1}, y_{2}, y_{3}\right)
$$

and $Y^{c}$ the set of contravariant components of $(Y)$ :

$$
Y^{c}=\left(y^{0}, y^{1}, y^{2}, y^{3}\right) \text {. }
$$

Here are some basic facts: the proofs follow the lines of [13] and can be found in Appendix C.

(i) For any given circle $U, V$

$$
\begin{aligned}
\Delta(U, V) & =G_{i j} u^{i} v^{j} \\
& =G^{i j} u_{i} v_{j} \\
& =u^{j} v_{j}=u_{j} v^{j} .
\end{aligned}
$$

(ii) If $\varepsilon^{1}, \varepsilon^{2}, \varepsilon^{3}, \varepsilon^{0}$ are the curvature of $Z_{1}, Z_{2}, Z_{3}, Z_{0}$, then the curvature $Y$ is given by

$$
\varepsilon_{Y}=y_{i} \varepsilon^{i}
$$

(iii) The polyspherical coordinate of $Z_{(i)}$ are the coordinates of the standard vector $e_{i}$ :

$$
Z_{(i) j}=\delta_{i j}
$$

We now specialize to the case where $Z_{i}=X_{i}$, where $X_{1}, X_{2}, X_{3}, X_{0}$ are the circles of the basic construction. In this case one verifies that there is a positive constant $\gamma>1$ so that

$$
\Delta\left(X_{i}, X_{j}\right)=G^{i j}=\gamma \text { for } i \neq j .
$$

In fact with $\alpha=\frac{\pi}{N}(N>6)$

$$
\gamma_{N}=\frac{1-2 \sin ^{2} \frac{\pi}{N}}{1-4 \sin ^{2} \frac{\pi}{N}}>1 .
$$

$\gamma$ reaches the value 1 for $N=\infty$ (the Apollonian case). 
The matrix $G$ can be rewritten:

$$
G=-(1+\gamma) I+\gamma J
$$

where $I$ is the unit matrix and $J$ is the matrix consisting of 1's. Since the spectrum of $\gamma J$ is $\{4 \gamma, 0,0,0\}$, we see that the spectrum of $G$ is

$$
\sigma(G)=\{3 \gamma-1,-(1+\gamma),-(1+\gamma),-(1+\gamma)\}
$$

Therefore the signature of the quadratic form associated to $\Delta$ will always be + $\ldots$. Our space will be the four dimensional Minkowski space. Relations (IV.14) tells us that:

$$
\Delta(U, U)=-1=u_{i} u^{i}
$$

Our circles are therefore space-like vectors of norm 1 in the Minkowski space. Therefore we move or transfer our Möbius maps $T_{1}, T_{2}, T_{3}$ to this four dimensional setting. (The Minkowski four dimensional space.)

Remark $I V .1 . \Delta(U, V)$ is clearly invariant under any Möbius transformations.

The basis: $\left(X_{0}\right),\left(X_{1}\right),\left(X_{2}\right),\left(X_{3}\right)$ is transformed by $T_{1}^{-1}$ into a new basis: $\left(X_{1}\right),\left(X_{\infty}\right),\left(X_{2}\right),\left(X_{3}\right)$. Clearly the $G$ matrix associated to this new basis is the same as the previous $G$ matrix. It is useful to compute the contravariant component of $\left(X_{\infty}\right)$ in the initial basis, an easy calculation gives:

$$
\begin{aligned}
& \Delta\left(X_{0}, X_{\infty}\right)=X_{(\infty)}^{0}=1+3 \gamma \tau, \\
& \Delta\left(X_{1}, X_{\infty}\right)=\gamma=X_{(\infty)}^{1}, \\
& \Delta\left(X_{2}, X_{\infty}\right)=\gamma=X_{\infty}^{(2)}, \\
& \Delta\left(X_{3}, X_{\infty}\right)=\gamma=X_{\infty}^{(3)},
\end{aligned}
$$

where

$$
\begin{aligned}
& \tau=\frac{2 \gamma}{2 \gamma-1}=2 \cos \frac{2 \pi}{N} \\
& \gamma=\frac{\tau}{2(\tau-1)}=\frac{2 \cos \frac{2 \pi}{N}}{2 \cos \frac{2 \pi}{N}-1}
\end{aligned}
$$

Proposition 1. There are matrices $M_{i}, i=1,2,3$ so that for any circle $Y$ :

$$
\left(T_{i} Y\right)_{c}=M_{i} Y_{c} \text {. }
$$

Proof.

$$
\left(T_{i} Y\right)_{c}=G^{-1}\left(T_{i} Y\right)^{c}=G^{-1}\left[\begin{array}{l}
\Delta\left(X_{0}, T_{i} Y\right) \\
\Delta\left(X_{1}, T_{i} Y\right) \\
\Delta\left(X_{2}, T_{i} Y\right) \\
\Delta\left(X_{3}, T_{i} Y\right)
\end{array}\right]
$$

and by invariance of the scalar product

$$
\Delta(U, T V)=\Delta\left(T^{-1} U, V\right),
$$




$$
\left(T_{i} Y\right)_{c}=G^{-1}\left[\begin{array}{c}
\Delta\left(T_{i}^{-1} X_{0}, Y\right) \\
\Delta\left(T_{i}^{-1} X_{1}, Y\right) \\
\Delta\left(T_{i}^{-1} X_{2}, Y\right) \\
\Delta\left(T_{i}^{-1} X_{3}, Y\right)
\end{array}\right]
$$

For clarity of the proof we specialize to $i=1$, choosing $T_{i}=T_{1}$ then

$$
\left(T_{1} Y\right)_{c}=G^{-1}\left(\begin{array}{c}
\Delta\left(X_{1}, Y\right) \\
\Delta\left(X_{\infty}, Y\right) \\
\Delta\left(X_{2}, Y\right) \\
\Delta\left(X_{3}, Y\right)
\end{array}\right)
$$

Each component of the column vector in (IV.30) is a linear combination of the $Y_{k}$, therefore $\left(T_{1}, Y\right)_{c}$ is of the form (IV.26).

We have to find the explicit form of the $M_{i}$. Let us for example, compute $M_{1}$. The columns of $M_{1}$ are $M_{1} e_{0}, M_{1} e_{1}, M_{1} e_{2}, M_{1} e_{3}$, from left to right. Now:

$$
\begin{aligned}
& T_{1} X_{1}=X_{0} \rightarrow M_{1} e_{1}=e_{0} \\
& T_{1} X_{2}=X_{2} \rightarrow M_{1} e_{2}=e_{2} \\
& T_{1} X_{3}=X_{3} \rightarrow M_{1} e_{3}=e_{3} \\
& T_{1} X_{0}=X_{1,0} \rightarrow M_{1} e_{0}=e_{1,0} .
\end{aligned}
$$

We only have to compute

$$
M_{1} e_{0}=\left(T_{1} X_{0}\right)_{c} .
$$

[Remember that (IV.14) tells us that the polyspherical coordinate of the basic circles are the basic vectors coordinates!]

$$
\left(T_{1} X_{0}\right)_{c}=G^{-1}\left(T_{1} X_{0}\right)^{c}
$$

and

$$
\left(T_{1} X_{0}\right)^{c}=\left[\begin{array}{c}
\Delta\left(X_{0}, T_{1}, X_{0}\right) \\
\Delta\left(X_{1}, T_{1}, X_{0}\right) \\
\Delta\left(X_{2}, T_{1}, X_{0}\right) \\
\Delta\left(X_{3}, T_{1}, X_{0}\right)
\end{array}\right]=\left[\begin{array}{c}
\Delta\left(X_{1}, X_{0}\right) \\
\Delta\left(X_{\infty}, X_{0}\right) \\
\Delta\left(X_{2}, X_{0}\right) \\
\Delta\left(X_{3}, X_{0}\right)
\end{array}\right],
$$

and finally using the fact that

$$
G^{-1}=-\frac{1}{1+\gamma}\left[I-\frac{\gamma}{3 \gamma-1} J\right] .
$$

Therefore we get

$$
\left(T_{1} X_{0}\right)_{c}=\left[\begin{array}{c}
\tau \\
-1 \\
\tau \\
\tau
\end{array}\right]
$$

or

$$
M_{1} e_{0}=\tau e_{0}-e_{1}+\tau e_{2}+\tau e_{3}
$$


and

$$
M_{1}=\left[\begin{array}{cccc}
\tau & 1 & 0 & 0 \\
-1 & 0 & 0 & 0 \\
\tau & 0 & 1 & 0 \\
\tau & 0 & 0 & 1
\end{array}\right]
$$

In the same way we get:

and

$$
\begin{aligned}
& M_{2} e_{0}=\tau e_{0}+\tau e_{1}-e_{2}+\tau e_{3}, \\
& M_{2} e_{1}=e_{1}, \\
& M_{2} e_{2}=e_{0}, \\
& M_{2} e_{3}=e_{3},
\end{aligned}
$$

$$
\begin{aligned}
& M_{3} e_{0}=\tau e_{0}+\tau e_{1}+\tau e_{2}-e_{3}, \\
& M_{3} e_{1}=e_{1}, \\
& M_{3} e_{2}=e_{2}, \\
& M_{3} e_{3}=e_{0} .
\end{aligned}
$$

Therefore

$$
M_{2}=\left[\begin{array}{cccc}
\tau & 0 & 1 & 0 \\
\tau & 1 & 0 & 0 \\
-1 & 0 & 0 & 0 \\
\tau & 0 & 0 & 1
\end{array}\right]
$$

and

$$
M_{3}=\left[\begin{array}{cccc}
\tau & 0 & 0 & 1 \\
\tau & 1 & 0 & 0 \\
\tau & 0 & 1 & 0 \\
-1 & 0 & 0 & 0
\end{array}\right]
$$

The eigenvalues of $M_{i}$ are: $1,1, e^{ \pm 2 i \pi / N}$. The eigenvectors corresponding to the eigenvalues 1 are trivial to find. The one corresponding to $e^{ \pm 2 i \pi / N}$ are for $M_{1}$ :

$$
-e^{ \pm i \pi / N} e_{0}+e^{\mp i \pi / N} e_{1} \pm \frac{i \cos \frac{2 \pi}{N}}{\sin \frac{\pi}{N}}\left(e_{2}+e_{3}\right)
$$

Remark. It is fundamental to notice that all these formulae and the isomorphism between the $M_{i}$ and $T_{i}$ make sense only for $N \geqq 7$. If one would extend the previous results to $N=2$ for instance, we should have $M_{i}^{2}=I$, however:

$$
M_{1}=\left[\begin{array}{cccc}
-2 & 1 & 0 & 0 \\
-1 & 0 & 0 & 0 \\
2 & 0 & 1 & 0 \\
2 & 0 & 0 & 1
\end{array}\right]
$$


and $M_{1}^{2} \neq I$ while $T_{1}^{2}=I$. The eigenvalues of $M$ are $1,1,-1,-1$ and only 3 eigenvectors exist in this case $e_{2}, e_{3}$ and $e_{0}+e_{1}-e_{2}-e_{3}$. Therefore the group generated by $M_{1}$ is infinite, being isomorphic to a translation group, while for $T_{1}$ it is finite of order 2 .

To further study why the analytic continuation of the two groups $\left\langle T_{1}, T_{2}, T_{3}\right\rangle$ and $\left\langle M_{1}, M_{2}, M_{3}\right\rangle$ which are isomorphic for $N \geqq 7$ do not continue to be isomorphic for $N \leqq 6$, one must go back to the fundamental quadratic form generated by $G$.

$$
X_{c}^{T} G X_{c}=-1 \text {. }
$$

Using (IV.18), we see that the eigenvalues of $G$ are such that the form is:

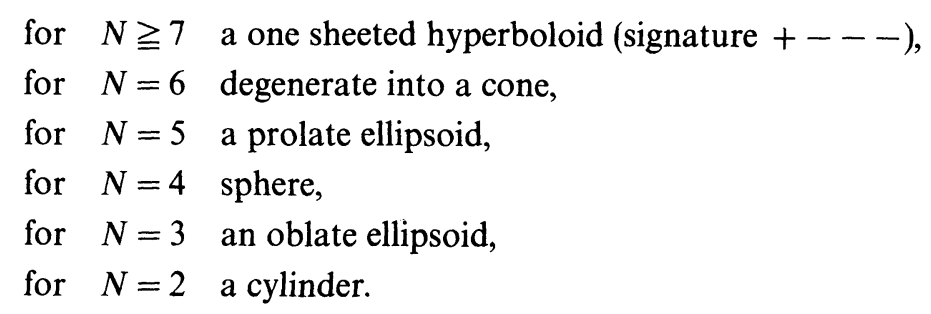

\section{Representation of the Matrix Elements of the Group in Terms of Tchebycheff Polynomials}

Clearly the group $\left\langle M_{1}, M_{2}, M_{3}\right\rangle$ is generated by two generators. For instance $M_{1}$ and $P$, where $P$ corresponds to the rotation $R$ for the group $\left\langle T_{1}, T_{2}, T_{3}\right\rangle . P$ is simply the permutation:

$$
P=(1,2,3)(0)
$$

The most general element of the group can therefore be written:

$$
M_{1}^{n_{1}} P M_{1}^{n_{2}} P M_{1}^{n_{3}} P \cdots
$$

It is therefore a product of elements of the form

$$
E_{n}=M_{1}^{n} P
$$

[We only need to consider non-negative powers $n$, because $M_{1}^{n}=I$.] Let us find the action of $E_{n}$. We have

$$
\begin{aligned}
& E_{n} e_{0}=M_{1}^{n} e_{0}, \\
& E_{n} e_{1}=e_{2}, \\
& E_{n} e_{2}=e_{3}, \\
& E_{n} e_{3}=M_{1}^{n} e_{1}=M_{1}^{n-1} M_{1} e_{1}=M_{1}^{n-1} e_{0} .
\end{aligned}
$$

Therefore it suffices to study $M_{1}^{n} e_{0}$ for $n \geqq 0$. We have the following result (see 
Appendix $\mathrm{C}$ for the proof). For $n \geqq 0$

$$
\begin{aligned}
M_{1}^{n} e_{0} & =e_{0} \frac{\sin \frac{2 \pi}{N}(n+1)}{\sin \frac{2 \pi}{N}}-e_{1} \frac{\sin \frac{2 \pi}{N} n}{\sin \frac{2 \pi}{N}}+\left(e_{2}+e_{3}\right) \frac{\cos \frac{2 \pi}{N} \sin (n+1) \frac{\pi}{N}}{\cos \frac{\pi}{N}} \frac{\sin \frac{n \pi}{N}}{\sin \frac{\pi}{N}} \frac{\sin \frac{\pi}{N}}{\sin } \\
& =q_{n} e_{0}-q_{n-1} e_{1}+r_{n}\left(e_{2}+e_{3}\right),
\end{aligned}
$$

where $q_{n}$ and $r_{n}$ are polynomials in $\tau$ with integer coefficients. Actually $q_{n}$ is the Tchebycheff polynomial of second kind $U_{k}\left(\frac{\tau}{2}\right)$.

Let us define a "word" $w$ as being a certain product

$$
w=E_{n_{1}} E_{n_{2}} \cdots E_{n_{k}} \text {. }
$$

The "distance" between two circles is:

$$
\Delta(X, Y)=\Delta\left(w_{1} X_{0}, w_{2} X_{0}\right),
$$

where $w_{1}, w_{2}$ are words made from the group, those words map $X_{0}$ onto $X$ and $X_{0}$ onto $Y$. Because of the invariance of $\Delta$ under any Möbius transformation

$$
\Delta(X, Y)=\Delta\left(X_{0}, w_{1}^{-1} w_{2} X_{0}\right)=\Delta\left(X_{0}, w X_{0}\right)
$$

If we want that no circles intersect we must have

$$
\left|\Delta\left(X_{0}, w X_{0}\right)\right| \geqq 1 .
$$

If furthermore we want to have all circles inside $X_{\infty}$, that is no circle inside $X_{0}$, we must have, either:

$$
\Delta\left(X_{0}, w X_{0}\right)=-1
$$

when $w X_{0} \equiv X_{0}$ or

$$
\Delta\left(X_{0}, w X_{0}\right) \geqq 1
$$

when $X_{0} \neq w X_{0}$. The equal sign occurs only for $N=\infty$. Combining (VII.11) and (VII.14) we have:

$$
\Delta\left(X_{0}, w X_{0}\right)=e_{0}^{T} G w e_{0}
$$

or writing

$$
w e_{0}=w_{0} e_{0}+w_{1} e_{1}+w_{2} e_{2}+w_{3} e_{3},
$$

we find

$$
\Delta\left(X_{0}, w X_{0}\right)=-w_{0}+\gamma\left(w_{1}+w_{2}+w_{3}\right)
$$

Clearly the $w_{i}$ being polynomials in $\tau$ and $\gamma$ being a rational in $\tau$, all elements here belong to cyclotomic fields.

Let us consider the next word after $w$ :

$$
w^{\prime}=E_{n} w
$$

Let us remark that we can consider any values $n=1,2, \ldots, N-1$ for $n$, because 
for $n=0, w^{\prime}$ and $w$ give the same $\Delta$. We now compute

$$
\begin{aligned}
& w_{0}^{\prime}=w_{0} q_{n}+w_{3} q_{n-1}, \\
& w_{1}^{\prime}=-\left[w_{0} q_{n-1}+w_{3} q_{n-2}\right], \\
& w_{2}^{\prime}=w_{1}+w_{0} r_{n}+w_{3} r_{n-1}, \\
& w_{3}^{\prime}=w_{2}+w_{0} r_{n}+w_{3} r_{n-1} .
\end{aligned}
$$

The $q_{n}$ are the Tchebycheff polynomials of the second kind and verify

$$
\begin{aligned}
q_{n} & =\tau q_{n-1}-q_{n-2}, \\
r_{n+1} & =r_{n}+\tau q_{n} .
\end{aligned}
$$

Let us apply the previous results to the case $N=\infty$. In this case we cannot take only $n$ positive, but for any $n \in \mathbb{Z}$, we have:

$$
\begin{aligned}
q_{n} & =n+1 \\
r_{n} & =n(n+1) \\
\gamma & =+1
\end{aligned}
$$

and (V.17) reads

$$
\begin{aligned}
& w_{0}^{\prime}=(n+1) w_{0}+n w_{3}, \\
& w_{1}^{\prime}=-\left[n w_{0}+(n-1) w_{3}\right], \\
& w_{2}^{\prime}=w_{1}+n(n+1) w_{0}+n(n-1) w_{3}, \\
& w_{3}^{\prime}=w_{2}+n(n+1) w_{0}+n(n-1) w_{3} .
\end{aligned}
$$

If we compute $\Delta^{\prime}-\Delta$ we get

$$
\Delta^{\prime}-\Delta=2 n\left[n w_{0}+(n-2) w_{3}\right] \text {. }
$$

The $w_{i}$ being integers the difference of any $\Delta_{w}$ and $\Delta_{w^{\prime}}$ has to be an even integer! Because $\Delta\left(X_{0}, X_{0}\right)=-1$, it then results that

$$
\Delta_{w}=\text { odd integer, }
$$

and therefore

$$
\left|\Delta_{w}\right| \geqq 1
$$

as it should. We have rederived here a well known result [13]. Inside the cyclotomic field, $\Delta$ is a finite sum of integers over the various components of the field. We shall explore in forthcoming papers this structure.

\section{Recovery of the Platonician Groups}

This case corresponds to $r^{2} \geqq \frac{9}{8}$, and therefore when $\alpha=\frac{\pi}{N}, N$ can only take the values $2,3,4,5$. From (II.8) the fixed points of $T_{1}$ are:

$$
f_{ \pm}=-\frac{1}{2} \pm \sqrt{r^{2}-\frac{3}{4}}=-\frac{1}{2} \pm \frac{\sqrt{3}}{2} \sqrt{\frac{1-\cos 2 \alpha}{1-3 \cos 2 \alpha}} \text {. }
$$


They are both real and $T_{1}$ is elliptic. The values of $r^{2}$ and $\alpha$ of interest are:

\begin{tabular}{|c|c|c|c|c|}
\hline$\alpha$ & $\frac{\pi}{5}$ & $\frac{\pi}{4}$ & $\frac{\pi}{3}$ & $\frac{\pi}{2}$ \\
\hline$r^{2}$ & $\frac{3(3+\sqrt{5})}{2}$ & $\frac{3}{2}$ & $\frac{6}{5}$ & $\frac{9}{8}$ \\
\hline
\end{tabular}

We used the fact that $\cos \frac{\pi}{5}=\frac{1+\sqrt{5}}{4}$ and $\cos \frac{2 \pi}{5}=\frac{\sqrt{5}-1}{4}$. Since

$$
f_{+} f_{-}=1-r^{2}<0
$$

we can find a dilation factor $d$ so that:

In fact

$$
\left(d f_{+}\right)\left(d f_{-}\right)=1
$$

$$
d=\sqrt{2(1-3 \cos 2 \alpha)} .
$$

Proposition 1. Let $d$ given by (VI.4) and define:

$$
\hat{T}_{i}=D T_{i} D^{-1} \quad \text { or } \quad \hat{T}_{i}(z)=d T_{1}\left(d^{-1} z\right) .
$$

Then $\left\langle\widehat{T}_{1}, \hat{T}_{2}, \hat{T}_{3}\right\rangle$ is a group of rotations of the Riemann sphere.

Proof. We first recall that the necessary and sufficient condition for an elliptic Möbius transformation to be a rotation of the Riemann sphere is that

$$
z_{f_{1}} z_{f_{2}}^{*}=-1
$$

where $z_{f_{1}}$ and $z_{f_{2}}$ are the fixed points of the transformation. It then follows that $\hat{T}_{1}$ is a rotation of the Riemann sphere. Now, the fixed points of $\hat{T}_{2}$ are $w d f_{ \pm}$and:

$$
\left(\omega d f_{+}\right)^{*}\left(\omega d f_{-}\right)=d f_{+} d f_{-}=-1 \text {. }
$$

So $\hat{T}_{2}$ is also a rotation (of the same angle $2 \alpha$ ), as is $\hat{T}_{3}$. Therefore $\hat{T}_{1}, \hat{T}_{2}, \hat{T}_{3}$ are three rotations of the same angle $2 \alpha$ of the Riemann sphere; we now determine the angles between the axes of rotation.

We represent the Riemann sphere, as a sphere of radius 1 , which intersects the complex plane in its center. If $P=\left(\frac{2 x}{|x|^{2}+1} ; \frac{2 y}{|z|^{2}+1} ; \frac{|z|^{2}-1}{|z|^{2}+1}\right)$ is the point on the Riemann sphere corresponding to $z=x+i y$ in the complex plane, and if $P_{\omega}$ and $P_{\omega^{2}}$ correspond respectively to $\omega z$ and $\omega^{2} z$, then:

$$
P P_{\omega}=P_{\omega} P_{\omega^{2}}=P_{\omega^{2}} P=\frac{\left(|z|^{2}-1\right)^{2}-2|z|^{2}}{\left(|z|^{2}+1\right)^{2}}=\cos \gamma \text {. }
$$

Computing, with $z=d f_{-}$, we get:

$$
\left|d f_{-}\right|=-d f_{-}=\frac{1}{\sqrt{2}}\{\sqrt{1-3 \cos 2 \alpha}+\sqrt{3} \sqrt{1-\cos 2 \alpha}\}
$$


and

$$
\cos \gamma=\frac{\cos 2 \alpha}{\cos 2 \alpha-1}
$$

where $\gamma$ is the common angle between the three axes of rotation corresponding to the rotations $\hat{T}_{1}, \hat{T}_{2}, \hat{T}_{3}$.

For our cases:

\begin{tabular}{|c|c|c|c|c|}
\hline$\alpha$ & $\frac{\pi}{2}$ & $\frac{\pi}{3}$ & $\frac{\pi}{4}$ & $\frac{\pi}{5}$ \\
\hline $\cos \gamma$ & $\frac{1}{2}$ & $\frac{1}{3}$ & 0 & $-\frac{1}{\sqrt{5}}$ \\
\hline
\end{tabular}
Proposition 2. For $\alpha=\frac{\pi}{4}$ and $\alpha=\frac{\pi}{2},\left\langle\hat{T}_{1}, \hat{T}_{2}, \hat{T}_{3}\right\rangle$ is conjugate to the rotation group
of a cube.

Proof. For $\alpha=\frac{\pi}{2},\left(\hat{T}_{i}\right)^{2}=1$ and the axes of rotation mutually meet at $60^{\circ}$. This is the case when the axes of rotation pass through the centers of two adjacent edges. See Fig. 5.

For $\alpha=\frac{\pi}{4}$, the axes of rotation are mutually orthogonal and pass through the centers of the faces of the cube.

For $N=4$, we also give in Fig. 6 , the set made of 4 circles which is invariant by $\left\langle T_{1}, T_{2}, T_{3}\right\rangle$.

Proposition 3. For $\alpha=\frac{\pi}{3}$ and $\alpha=\frac{\pi}{5},\left\langle\hat{T}_{1}, \hat{T}_{2}, \hat{T}_{3}\right\rangle$ is conjugate to the rotation group of an icosahedron or dodecahedron.

Proof. Again, the angles are right. For $\alpha=\frac{\pi}{5}$ we consider a dodecahedron; the axes of rotation pass through the centers of three pentagons that share a common

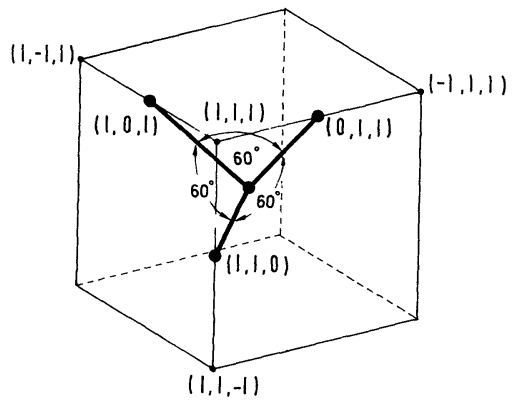

Fig. 5. The case $N=2$ 


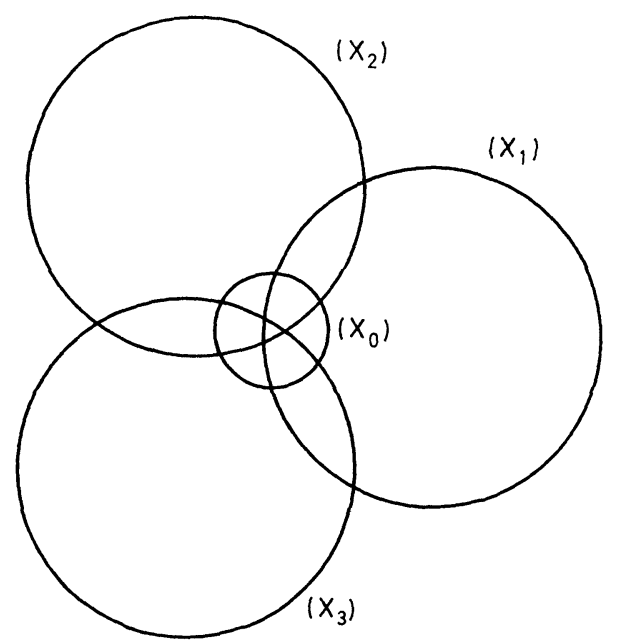

Fig. 6. The case $N=4$

vertex. For $\alpha=\frac{\pi}{3}$, we consider an icosahedron; the axes of rotation pass through the centers of three triangles that are adjacent to a single common triangle.

Remark 1. For $N=2,3,4,5$ we can also consider $\alpha=\frac{k \pi}{N}$, we then discover that we obtain nothing new. The result is obvious for $N=2,3,4$. For $N=5$ one could think that $\alpha=\frac{\pi}{5}$ and $\alpha=\frac{2 \pi}{5}$ would give different results, however computing $\cos \gamma$, one finds $\cos \gamma=-\frac{1}{\sqrt{5}}$ for $\alpha=\frac{\pi}{5}$ and $\cos \gamma=+\frac{1}{\sqrt{5}}$ for $\alpha=\frac{2 \pi}{5}$. This shows that the axes of rotations are in fact the same in both cases.

Remark 2. One should be able to prove that the only cases where a finite group is generated is for $N=2,3,4,5$.

\section{The Case $\alpha=\frac{\pi}{6}$}

We come now to the case $\alpha=\frac{\pi}{6}$. The fixed point of $T_{1}$ are, making use of (II.8) and (II.9): $\omega$ and $\omega^{2}$. So the fixed points of $T_{2}$ are $\omega^{2}$ and 1 and those of $T_{3}$ are 1 and $\omega$.

Let us send:

$$
\left\{\begin{aligned}
\omega & \rightarrow \infty \\
\omega^{2} & \rightarrow 0 \\
1 & \rightarrow \omega^{2}
\end{aligned}\right.
$$


with the transformation:

define

$$
\left\{\begin{aligned}
f(z) & =-\frac{z-\omega^{2}}{z-\omega} \\
f^{-1}(z) & =\omega \frac{(z+\omega)}{z+1}
\end{aligned}\right.
$$

$$
\tilde{T}_{i}=f \circ T_{i} \circ f^{-1} \text {. }
$$

So

$$
\begin{aligned}
& \tilde{T}_{1} z=e^{2 i \pi / 6} z, \\
& \tilde{T}_{2} z=-\omega \frac{z}{z+1}, \\
& \tilde{T}_{3} z=e^{-2 i \pi / 6} z-\omega .
\end{aligned}
$$

The group $\left\langle\widetilde{T}_{1}, \widetilde{T}_{3}\right\rangle$ is a classical doubly periodic group, whose general element has the form

$$
S z=z e^{2 i \pi n / 6}+m_{+} e^{2 i \pi / 6}+m_{-} e^{-2 i \pi / 6} ; \quad m_{+}, m_{-} \in \mathbb{Z}, \quad n=1,2,3,4,5,6 .
$$

The only limit point is $\infty \mathrm{cf}$. [8].

Claim. The group $\left(\tilde{T}_{1}, \tilde{T}_{2}, \tilde{T}_{3}\right)$ has $\tilde{\mathbb{C}}$ as its limit and is thus not discontinuous.

Clearly the lattice is not invariant by $\widetilde{T}_{2}$ because

$$
\tilde{T}_{2} z=-\frac{\omega z}{z+1}=-\omega+\frac{\omega}{z+1}
$$

while the various transformations $z \rightarrow z+1, z \rightarrow \omega z, z \rightarrow z-\omega$ leave the lattice invariant, the transformation $z \rightarrow \frac{1}{z}$ does not.

\section{Higher Dimensional Extensions}

One can try to extend the method introduced here to higher dimensions by starting with $n+1 n$-dimensional spheres $X_{1}, \ldots, X_{n}$ with centers at the vertices of a regular simplex in $\mathbb{R}^{n}$ and looking for a sphere $X_{0}$ and conformal maps $T_{1}, \ldots, T_{n}$ satisfying $T_{i} X_{i}=X_{0}, T_{i} X_{j}=X_{j}$ for $j \neq i$. In $\mathbb{R}^{3}$ the spheres $X_{0}, X_{1}, \ldots, X_{4}$ turn out to be mutually tangent and a packing investigated by Boyd [13] is the result. In this case $T_{i}^{6}=I$. In $\mathbb{R}^{4}$ we believe that there is a non-tangential packing generated by maps that satisfy $T_{i}^{5}=I$. We believe that these exhaust the finite dimensional packings that can be constructed with this technique. There is, however, an infinite dimensional packing generated by maps satisfying $T_{i}^{4}=I$. Details of these constructions will appear elsewhere.

\section{Conclusion}

We have studied a class of discrete subgroups of $S L(2, \mathbb{C})$ generated by two rotations. These subgroups fall into two classes: finite and infinite. The finite 
subgroups give the symmetry groups of the cube and icosahedron. The infinite subgroups have as their limit sets packings of circles. The action of the infinite groups can be analyzed in a four-dimensional Minkowski space and some preliminary analysis was made.

The next investigations will be to analyse the structure of the lattice on the one sheeted Minkowski hyperboloid, in particular its projection on the three dimensional Euclidean space. Also the generalization to higher dimensions, as well as the construction of discrete groups depending on more than one parameter, will be analyzed.

\section{Appendix A}

Denote the center and radius of the circle of inversion by $-I$ and $k$ respectively.

The circle of inversion $J$ and $X_{2}$ being by construction orthogonal at $\xi_{2}$ (see Fig. 1), we have:

$$
r^{2}+k^{2}=\frac{3}{4}+\left(I-\frac{1}{2}\right)^{2}
$$

For real $x$, we must have:

$$
(J x+I)(x+1)=k^{2} .
$$

For the center of $\left(X_{0}\right)$ to be at 0 , we force, (using $\left.J X_{1}=S X_{0}\right)$ :

$$
\frac{J(1+r)+J(1-r)}{2}=-1 \text {. }
$$

From these we obtain:

$$
I=\frac{1 \pm \sqrt{9-8 r^{2}}}{2}
$$

We take the "+" sign. Later we will remark on the other choice of sign. We also have:

$$
J z=\left\{\begin{array}{l}
\frac{k^{2}(x+I)}{(x+I)^{2}+y^{2}}-I \\
\frac{k^{2} y}{(x+I)^{2}+y^{2}}
\end{array} \text { and } S z=\left\{\begin{array}{l}
-(1+x) \\
y
\end{array},\right.\right.
$$

where the upper line refers to the real part, while the lower one to the imaginary. With

$$
I=\frac{1+\sqrt{9-8 r^{2}}}{2}
$$

we derive from (II.5) and (A.5):

$$
T_{1} z=\frac{I-1}{2} \frac{2 z-I}{z+I}
$$


From (A.1) and (A.6) we obtain the radius of the circle of inversion $k$ :

$$
k^{2}=\left(I-\frac{1}{2}\right)^{2}+\frac{3}{4}-r^{2}=3\left(1-r^{2}\right) \text {. }
$$

One also checks that:

$$
\rho_{0}=\frac{k^{2} r}{(I+1)^{2}-r^{2}}=\frac{2 r^{2}-3+\sqrt{9-8 r^{2}}}{2 r},
$$

or in invariant form

$$
\rho_{0}=\frac{\sqrt{\cos 2 \alpha+1} \sqrt{3 \cos 2 \alpha-1}-\sqrt{3} \cos 2 \alpha}{\sqrt{2(2 \cos 2 \alpha-1)(3 \cos 2 \alpha-1)}} .
$$

In the same way:

$$
\rho_{\infty}=\frac{k^{2} r}{r^{2}-(2-I)^{2}}=\frac{\sqrt{\cos 2 \alpha+1} \sqrt{3 \cos 2 \alpha-1}+\sqrt{3} \cos 2 \alpha}{\sqrt{2(2 \cos 2 \alpha-1)(3 \cos 2 \alpha-1)}} .
$$

\section{Appendix B}

We are to prove that for $k \geqq 2$ and $0 \leqq \xi \leqq \frac{\pi}{6 k+1}, k \in \mathbb{N}$, we have

$$
\frac{1-4 \sin ^{2} k \xi}{1-3 \sin ^{2} k \xi} \cdot \frac{\sin ^{2} k \xi}{\sin ^{2} \xi}>1
$$
With $\chi=\sin ^{2} k \xi$ we have $\sin \xi=\sin \left\{\frac{\sin ^{-1} \sqrt{\chi}}{k}\right\}$ and the following inequality
equivalent to B.1:

$$
\frac{\sin ^{-1} \sqrt{\chi}}{\sin ^{-1}\left(\sqrt{\frac{\chi(1-4 \chi)}{1-3 \chi}}\right)}<k, \quad 0 \leqq \chi \leqq \sin ^{2}\left(\frac{\pi}{6+\frac{1}{k}}\right)
$$
Now, for $u \geqq 0, \sin u \leqq u$ so $u \leqq \sin ^{-1} u$. Also, for $0 \leqq u \leqq 1 / 2, \frac{\sin ^{-1} u}{u}<\frac{2}{\sqrt{3}}$ by
the Mean Value Theorem. This gives

$$
\frac{\sin ^{-1} \sqrt{\chi}}{\sin ^{-1}\left(\sqrt{\frac{\chi(1-4 \chi)}{1-3 \chi}}\right)} \leqq \frac{\sin ^{-1} \sqrt{\chi}}{\sqrt{\chi}} \sqrt{\frac{1-3 \chi}{1-4 \chi}}<\frac{2}{\sqrt{3}} \sqrt{\frac{1-3 \chi}{1-4 \chi}}
$$

for $0 \leqq \chi \leqq \sin ^{2}\left(\frac{\pi}{6+\frac{1}{k}}\right)<\frac{1}{4}$ 
Since $\frac{1-3 \chi}{1-4 \chi}$ is increasing, we see that it suffices to show that

$$
\frac{1-3 \sin ^{2}\left(\frac{\pi}{6+\frac{1}{k}}\right)}{1-4 \sin ^{2}\left(\frac{\pi}{6+\frac{1}{k}}\right)} \leqq \frac{3}{4} k^{2}
$$

or

$$
\sin ^{2}\left(\frac{\pi}{6+\frac{1}{k}}\right) \leqq \frac{1}{12}\left\{3-\frac{1}{k^{2}-1}\right\}
$$

Using

$$
\begin{aligned}
\sin ^{2} \frac{\pi}{6+\frac{1}{k}} & =\frac{1}{2}\left[1-\cos \frac{2 \pi}{6+\frac{1}{k}}\right] \\
\frac{1}{2} & =\cos \frac{\pi}{3}
\end{aligned}
$$

and

$$
\cos p-\cos q=2 \sin \left(\frac{p+q}{2}\right) \sin \left(\frac{q-p}{2}\right) .
$$

We see that (B.5) is equivalent to

and that

$$
\cos \frac{2 \pi}{6+\frac{1}{k}}-\frac{1}{2} \geqq \frac{1}{6} \cdot \frac{1}{k^{2}-1}
$$

$$
\cos \frac{2 \pi}{6+\frac{1}{k}}-\frac{1}{2} \geqq 2 \sin \left\{\frac{25 \pi}{78}\right\} \sin \left\{\frac{\pi}{36 k+6}\right\} \text {. }
$$

Now,

$$
\sin \frac{\pi}{36 k+6}>\frac{\pi}{36 k+6} \cdot \cos \frac{\pi}{78}
$$

since $\frac{\sin \chi}{\chi}=\cos \eta$ for some $0<\eta<\chi$ and $0<\frac{\pi}{36 k+6}<\frac{\pi}{28}$ for $k \geqq 2$.

Therefore,

$$
\cos \frac{2 \pi}{6+\frac{1}{k}}-\frac{1}{2} \geqq 2 \sin \frac{25 \pi}{78} \cos \frac{\pi}{78} \cdot \frac{\pi}{36 k+6}>\frac{5.2}{36 k+6}=\frac{1}{6} \cdot \frac{5.2}{6 k+1} .
$$


Finally, the function $f(z)=\frac{6 z+1}{z^{2}-1}$ is decreasing for $z \geqq 2$, so $\frac{5.2}{6 k+1} \geqq \frac{1}{k^{2}-1}$ for
$k \geqq 2$.

\section{Appendix C}

With $X=S(a, r)$ and $Y=S(b, s)$ as in (IV.1) we define

Then,

$$
v(Y)=\frac{1}{s}\left[\begin{array}{c}
|b|^{2}-s^{2} \\
\frac{1}{2} \\
\operatorname{Re} b \\
\operatorname{Im} b
\end{array}\right] \text { and } u(X)=\frac{1}{r}\left[\begin{array}{c}
\frac{1}{2} \\
|a|^{2}-r^{2} \\
-\operatorname{Re} a \\
-\operatorname{Im} a
\end{array}\right]
$$

$$
\Delta(X, Y)=v(Y)^{T} u(X) .
$$

From this we obtain the Darboux-Frobenius formula: if $X_{1}, X_{2}, X_{3}, X_{4}, X_{5}$ and $Y_{1}, Y_{2}, Y_{3}, Y_{4}, Y_{5}$ are circles in $\mathbb{R}^{2}$, then

$$
\operatorname{det}\left(\Delta\left(X_{i}, Y_{j}\right)\right)=0 .
$$

This is because $\Delta\left(X_{i}, Y_{j}\right)=v\left(Y_{j}\right)^{T} u\left(X_{i}\right)$ and $u\left(X_{1}\right), \ldots, u\left(X_{5}\right)$ are linearly dependent in $\mathbb{R}^{4}$.

Now take $X_{1}=Y_{1}=Z_{0}, X_{2}=Y_{2}=Z_{1}, X_{3}=Y_{3}=Z_{2}, X_{4}=Y_{4}=Z_{3}$ and $X_{5}=Z, Y_{5}=Y$. (C.3) becomes (cf. IV.6)

$$
\operatorname{det}\left[\begin{array}{cc}
G & \left(Y^{c}\right)^{T} \\
Z^{c} & \Delta(Y, Z)
\end{array}\right]=0
$$

By expansion

$$
\Delta(Y, Z) \operatorname{det} G-\left(Y^{c}\right)^{T}(\operatorname{adj} . G) Z^{c}=0, \quad \text { adj. } G=G^{-1} \operatorname{det} G .
$$

If $\operatorname{det} G \neq 0$, we obtain

$$
\Delta(Y, Z)=\left(Y^{c}\right)^{T} G^{-1} Z^{c}
$$

which establishes (IV.12). Assertions (IV.13-IV.14) follow from (IV.9).

For example, we see immediately that

$$
\left(Y^{c}\right)^{T} G^{-1}\left(Y^{c}\right)=Y_{c} G Y_{c}=-1 .
$$

Assertion (IV.16) is a trivial consequence of the definition (IV.9).

For assertion (IV.15), assume $Y=S(b, s)$ and that $Z$ is a line whose distance from $b$ is $d$ and whose distance from the center of $Z_{i}$ is $d_{i}$. Then, the extension of (IV.2) to the case of lines gives

where

$$
\Delta(Y, Z)=d \eta
$$

$$
\eta=\frac{1}{s}
$$

and

$$
\Delta\left(Z, Z_{i}\right)=d_{i} \varepsilon_{i} \quad \text { (without summation), }
$$


where

$$
\varepsilon_{i}=\text { curvature of } Z_{i}=\frac{1}{\text { radius of } Z_{i}}
$$

Now,

$$
d \eta=\Delta(Y, Z)=\left(Y^{c}\right)^{T} G^{-1} Z^{c}
$$
Letting $Z \rightarrow \infty$ we see that $\underset{d_{i}}{Z} \rightarrow 1$. So, the $d$ on the left of (C.12) can cancel with
each $d_{i}$ in $Z^{\mathrm{c}}$ yielding

$$
\eta=\left(Y^{c}\right)^{T} G^{-1}\left[\begin{array}{c}
\varepsilon_{0} \\
\varepsilon_{1} \\
\varepsilon_{2} \\
\varepsilon_{3}
\end{array}\right],
$$

which is what (IV.15) says.

\section{Appendix D}

The proof proceeds by induction on $n$. When $n=0$, we obtain $M_{1}^{0} e_{0}=e_{0}$. For $n=1$,

$$
M_{1} e_{0}=\tau e_{0}-e_{1}+\left(e_{2}+e_{3}\right) \tau \text {. }
$$

If we assume

$$
M_{1}^{k} e_{0}=q_{k}(\tau) e_{0}+p_{k}(\tau) e_{1}+r_{k}(\tau)\left(e_{2}+e_{3}\right)
$$

we will discover that

$$
\begin{aligned}
& p_{k+1}(\tau)=-q_{k}(\tau) \\
& q_{k+1}(\tau)=\tau q_{k}(\tau)+q_{k-1}(\tau) \\
& r_{k+1}(\tau)=r_{k}(\tau)+\tau q_{k}(\tau)=\sum_{j=0}^{k} \tau q_{j}(\tau)
\end{aligned}
$$

Since $q_{0}=1$ and $q_{1}(\tau)=\tau$, we see that

$$
q_{k}(\tau)=U_{k}\left(\frac{\tau}{2}\right)
$$

where

$$
U_{k}(\chi)=\frac{\sin (k+1) \theta}{\sin \theta}, \quad \chi=\cos \theta,
$$

are the Chebyshev polynomials of the second kind.

Standard manipulation now gives (V.5).

Acknowledgements. We thank Professor C. Itzykson for suggesting the connection to the Platonician groups. 


\section{References}

1. Mandelbrot, B.: The fractal geometry of nature. Oxford: Freeman 1984

2. Falconer, K. J.: The geometry of fractal sets. Cambridge: Cambridge University Press 1985

3. Adler, P. M.: Transport processes in fractals I, Int. J. Multiphase Flow 11, 91-108 (1985)

4. Bear, J.: Dynamics of fluids in porous media. Oxford: Dover 1988

5. Katz, A. J., Thompson, A. H.: Fractal sandstone pores: implications for conductivity pore formation. Phys. Rev. Lett. 54, 1325-1328 (1985)

6. Jaric, M. V.: Introduction to quasi-crystals. New York: Academic Press 1988

7. Katz, A.: Matching rules for the 3-dimensional Penrose tilings. In: Quasi-crystalline Materials. Janot, Ch., Dubois, J. M. (eds.). Singapore: World Scientific 1989

8. Ford, L. R.: Automorphic functions. Oxford: Chelsea 1929

9. Boyd, D. W.: The residual set dimension of the Apollonian packing. Mathematika 20, 170-174 (1973)

10. Tricot, C.: A new proof for the residual set dimension of the Apollonian packing. Math. Proc. Camb. Phil. Soc. 96, 413-423 (1984)

11. Kasner, E., Supnick, F.: The Apollonian packing of circles. Proc. Nat. Acad. Sci. USA 29, 378-384 (1943)

12. Berger, M.: Geometry II. Berlin, Heidelberg, New York: Springer 1987

13. Boyd, D. W.: The osculatory packing of a three-dimensional sphere. Can. J. Math. 25, 303-322 (1973)

Communicated by A. Connes 
